\title{
The significance of detailed structure in the boundary layer to thermal radiation at the surface in climate models
}

\author{
Wenzhong Zhao, ${ }^{1}$ William R. Kuhn and S. Roland Drayson \\ Department of Atmospheric, Oceanic and Space Sciences, University of Michigan, Ann Arbor
}

\begin{abstract}
This study used a mid-latitude measurement of diurnal variations in the boundary layer to simulate thermal radiation for a clear sky. The emissivity growth method was used for the absorption by the five major greenhouse gases averaged over $10 \mathrm{~cm}^{-1}$ from line by line calculations. The vertical resolution of the model was $0.6 \mathrm{mb}$ at the ground surface, and gradually increases to $100 \mathrm{mb}$ in the middle troposphere. The importance to the calculated surface and outgoing infrared radiation due to detailed boundary layer structure and diurnal variations is demonstrated. Thus there is a need to carefully consider the near surface atmosphere in construction of radiation models for use in climate studies.
\end{abstract}

\section{Introduction}

It has long been recognized that the lowest $200-300$ meters of the atmosphere is very important to the surface energy balance. Temperatures and water vapor mixing ratios change rapidly with height and time in this layer. The daily variation of surface air temperature ranges from less than $0.5 \mathrm{~K}$ to greater than 50 $\mathrm{K}$ and depends on the radiation energy budget, surface moisture and texture, and weather conditions. The daily variation is smaller than $0.5 \mathrm{~K}$ for an open deep sea, but greater than $2 \mathrm{~K}$ for shallow water. For moist soil, the daily variation is about $5-10 \mathrm{~K}$, but for dry sand it might be greater than $50 \mathrm{~K}$. Vegetation can have an appreciable effect on surface air temperature but is not included in this analysis.

Most current climate modeling places little emphasis on the thermal radiation with vertical resolution less than 10 millibar. A typical thickness in climate models is, for example, in the NCAR CCM0 or CCM1, about 20 millibars, i.e., about 160 meters in the lowest layer [Fels, 1991]. While there have been a few studies that include the question of the vertical resolution of the atmosphere in radiation calculations [Morcrette and Fouquart, 1985; Tjemkes et al, 1990, Ellingson et al, 1991; Feigelson et al, 1991; Fels et al, 1991], the importance of the bound-

\footnotetext{
${ }^{1}$ Now at Applied Research Corporation, NASA Goddard Space Flight Center, Code 913, Greenbelt, MD 20771
}

Copyright 1994 by the American Geophysical Union.

Paper number 94GL01393

0094-8534/94/94GL-01393\$03.00 ary layer to radiation modeling for surface energy budget is getting more and more attention. The questions addressed in this paper are, "How much error is generated in the downward infrared flux at the surface by not taking into account the detailed structure in the boundary layer of temperature and greenhouse gases and not including the diurnal variation? Is it adequate to use constant lapse rate profiles of temperature and/or greenhouse gases in the boundary layer to calculate the radiation fluxes for daily or long term mean values?"

\section{Diurnal Variations in the Boundary Layer}

There are relatively few detailed measurements of boundary layer diurnal temperatures and water vapor amounts. Among the more notable were O'Neill, Nebraska, USA, in 1953; and Hay, Australia, in 1967. Significant efforts in experimental research were also reported in Germany, Netherlands, and the former USSR. The diurnal variation of temperature used in this study was adopted from the measurements of Vorontsov made in September, 1956 at the Makhtaly Settlement (in the south of the former USSR) [Gol'tsberg, 1969], and located at the boundary between the semidesert and developed irrigated lands of the Golodnaya Steppe (Latitude $40.5^{\circ} \mathrm{N}$, Longitude $68.5^{\circ} \mathrm{E}$ ).

These measurements were chosen for several reasons. First, they were made under conditions for which there will be significant diurnal variations. The insolation at Makhtaly in September was large, and solar radiation produced a strong surface heating around early afternoon. The sky was clear, the soil moisture and air humidity were low, and the nocturnal cooling was intensive. The surface temperature dropped $19.5 \mathrm{~K}$, and a temperature inversion of greater than $12 \mathrm{~K}$ with thickness of about 500 meters developed by early morning. Secondly, these measurements were made at levels up to 300 meters every two hours, and they are a good representation of daily variations in the boundary layer.

The profile above 900 millibars was assumed not to change with time and was chosen to be identical with the standard profile for 45 degree north latitude in summer. A slight adjustment was made to fit the profiles smoothly. The temperature profiles used in the simulation are shown in Figure 1. 


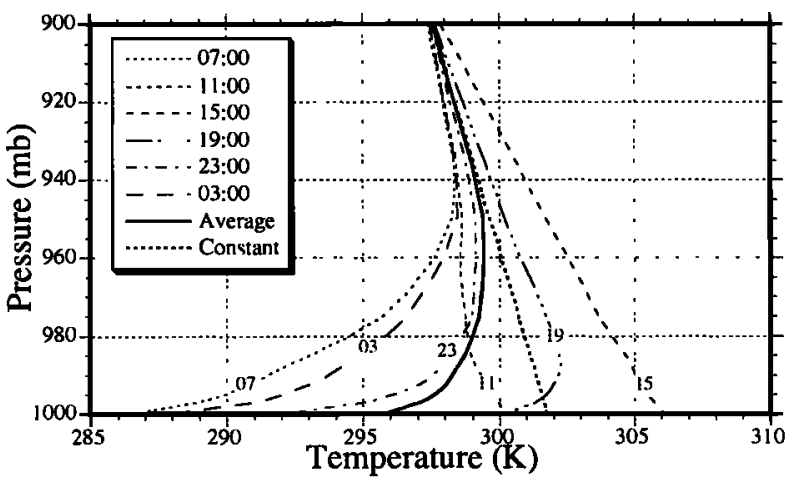

Figure 1. The 24 Hour Variation of Temperature in the Boundary Layer. Numbers indicate time. "Average" indicates the daily mean profile. "Constant" indicates a constant lapse rate profile.

The air temperature at the ground surface was extrapolated from the 2 meter height down to the surface, and the ground surface temperature was assumed the same as the air. The 24 hour variation of surface temperature is shown in Figure 2. The highest surface temperature occurred at $1500 \mathrm{hr}$, while the lowest occurred at $0700 \mathrm{hr}$.

Since no direct measurements of humidity were available, a standard relative humidity for 45 degree north latitude in summer was used to calculate the maximum water vapor mixing ratio profile corresponding to the maximum temperature profile at $1500 \mathrm{hr}$. In the cooling phase (from $1500 \mathrm{hr}$ to $0700 \mathrm{hr}$ ), water vapor mixing ratio in a layer was assumed not to change if the temperature in that layer was higher than the dewpoint corresponding to the maximum mixing ratio. When the temperature dropped below the dewpoint, the water vapor mixing ratio in that layer was assumed to be the saturation ratio at that temperature. It was also assumed that condensation occurred only at the ground surface and no fog formed in the air, because the experiment reported a clear sky. In the heating phase (from $0700 \mathrm{hr}$ to $1500 \mathrm{hr}$ ), the water vapor mixing ratio in a layer would increase to conform to the standard relative humidity profile for $\mathbf{4 5}$ degree north latitude in summer. The 24 hour variation of the mixing ratio used in these simulations is shown in Figure 3. At $1500 \mathrm{hr}$, the water vapor mixing ratio at the surface had its maximum of about $2.40 \times 10^{4} \mathrm{ppm}$; and at $0700 \mathrm{hr}$, a minimum about $9.50 \times 10^{3} \mathrm{ppm}$.

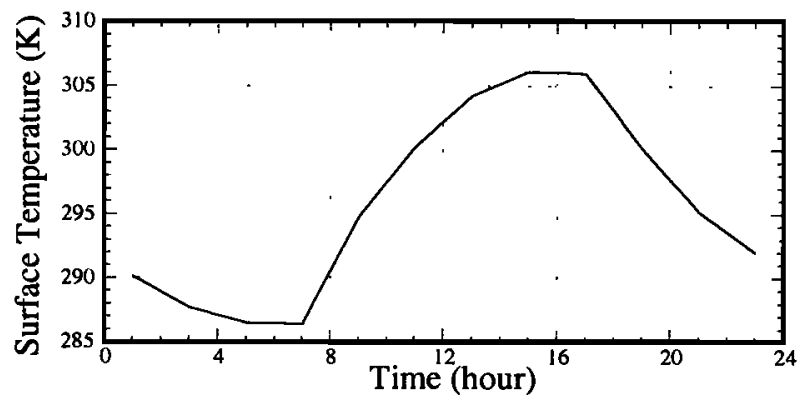

Figure 2. The 24 Hour Variation of the Surface Temperature.

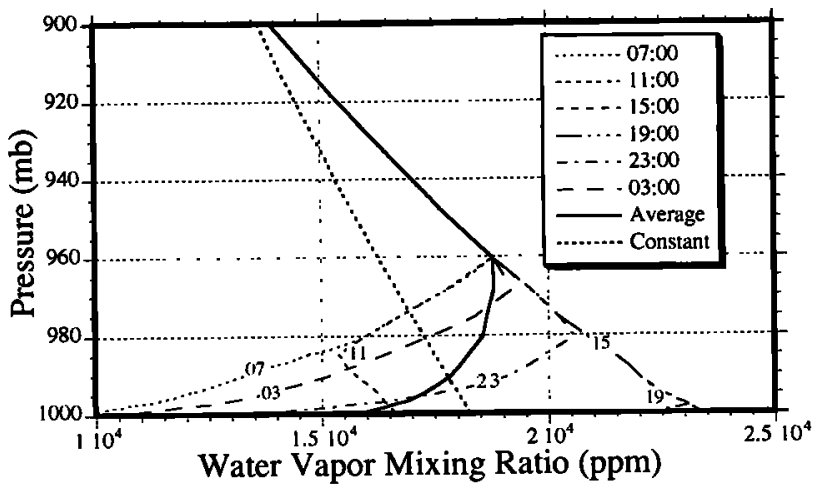

Figure 3. The 24 Hour Variation of $\mathrm{H}_{2} \mathrm{O}$ Mixing Ratio in the Boundary Layer. Numbers indicate time in hours. "Average" indicates the daily mean profile. "Constant" refers to the profile calculated from the constant temperature lapse rate profile (see Figure 1).

The constant lapse rate temperature profile is also shown in Figure 1. The average profile has an inversion near the surface with inversion magnitude of about 3.5 $\mathrm{K}$, and with the highest daily average temperature at about 300 meters (960 millibars). The constant lapse rate profile has a surface temperature that differs by $6.0 \mathrm{~K}$ from the 24 hour mean value; the constant lapse rate profile is closer to the daytime average than to the daily average. Observations showed that even in summer, the period of surface net radiation heating in midlatitude is only about 8 hours per day. The longer cooling phase and stronger surface radiation cooling cause the daily averaged temperature profile to have an inversion in the lower part of the boundary layer. The constant lapse rate profile does not adequately reflect the complicated clear sky air-land interaction, specifically, the characteristics of the nighttime profiles which have an inversion produced by strong nocturnal radiation cooling at the surface.

\section{Parameterization of Thermal Radiation Transfer}

A high spectral and vertical resolution infrared radiation transfer program was developed to simulate thermal radiation transfer in the atmosphere with emphasis on the planetary boundary layer. The emissivity growth method [Gordley et al., 1981; Weinreb, 1976] was used to calculate transmittances along inhomogeneous atmospheric paths. This method offers a combination of a fast and efficient algorithm, a high accuracy, and flexibility. The high accuracy is obtained by calculating the atmospheric transmittance database using the line-by-line method. Because this method does not require a priori scaling for pressure and temperature, the time consuming line-by-line computing needs to be done only once to establish an atmospheric transmittance database of fine band width, for example, 1 $\mathrm{cm}^{-1}$. It is flexible and easy to meet different requirements of accuracy and speed of computing by changing the width of bands and establishing a new database by simple averaging. 
This research included all absorption lines in the HITRAN86 database in the spectral region from 0 to $2800 \mathrm{~cm}^{-1}$ of five major greenhouse gases: $\mathrm{H}_{2} \mathrm{O}, \mathrm{CO}_{2}$, $\mathrm{O}_{3}, \mathrm{~N}_{2} \mathrm{O}$, and $\mathrm{CH}_{4}$. The overlap of absorption by different molecules is accounted for by multiplication of their individual transmittances. Water vapor continuum absorption was included; the broadening coefficient database calculated by Q. Ma (1991) was adopted.

The highest vertical resolution was near the surface where the thickness of the lowest 5 layers was $0.6 \mathrm{mb}$ (about 5 meters). Above $3 \mathrm{mb}$ and extending to 550 mb the thickness of each layer was $27 \%$ larger than the layer below it. Above $550 \mathrm{mb}$ the layer thickness was $100 \mathrm{mb}$.

The upward and downward thermal radiation fluxes at level $l, F^{\uparrow}(l)$ and $F^{\downarrow}(l)$, are numerically calculated by

$$
\begin{aligned}
& F^{\dagger}(l)=2 \pi \Delta \nu \sum_{k=1}^{K} \sum_{j=1}^{5}\left\{B\left(\nu_{k}, T_{s}\right) \pi_{l, n}\left(\xi_{\jmath}\right)\right. \\
& \left.+\sum_{\imath=l+1}^{n} B\left(\nu_{k}, T_{\imath}\right)\left[\epsilon_{l, 2}\left(\xi_{\jmath}\right)-\epsilon_{l, \imath-1}\left(\xi_{\jmath}\right)\right]\right\} \xi_{\jmath} w_{\jmath}, \\
& \text { for } l=0,1, \ldots, n-1 \text {; } \\
& F^{\uparrow}(n)=\pi \Delta \nu \sum_{k=1}^{K} B\left(\nu_{k}, T_{s}\right) \\
& F^{\downarrow}(0)=0 \text {; } \\
& F^{\downarrow}(l)=2 \pi \Delta \nu \sum_{k=1}^{K} \sum_{j=1}^{5} \sum_{\imath=1}^{l} B\left(\nu_{k}, T_{\imath}\right)\left[\epsilon_{\imath-1, l}\left(\xi_{\jmath}\right)\right. \\
& \left.-\epsilon_{\imath, l}\left(\xi_{3}\right)\right] \xi_{3} w_{3}, \quad \text { for } l=1,2, \ldots, n ;
\end{aligned}
$$

where subscripts $i, j, k$ are indexes of level (layer), slant path, and wavenumber, respectively. The whole atmosphere was divided into $n-1$ layers, and the index of the level is 0 at the top of the atmosphere, while it is $n$ at the surface; between level $l$ and level $l+1$ is layer l. $\Delta \nu$ is the band width of the transmittance database ( $10 \mathrm{~cm}^{-1}$ in this application), $\left.B_{(} \nu_{k}, T_{i}\right)$ is the Planck function at wavenumber $\nu_{k}$ and layer average temperature $T_{i}, \xi_{j}$ is the Gaussian abscissa or the cosine of zenith angle, $\tau_{l, n}\left(\xi_{j}\right)$ is the transmittance between level $l$ and $n$ and along the inhomogeneous path in the $\xi_{j}$ direction, $\epsilon_{i, l}\left(\xi_{j}\right)=1-\tau_{i, l}\left(\xi_{j}\right)$ is the emissivity between level $i$ and $l$ along the inhomogeneous path in the $\xi_{j}$ direction, and $w_{j}$ is the Gaussian quadrature weighting function. $T_{s}$ is the temperature of the ground surface, and the surface is assumed a black body, $\epsilon_{s}=1$.

\section{Results}

The calculated infrared fluxes are shown in Table

\begin{tabular}{|c|c|c|c|c|c|}
\hline \multirow[b]{2}{*}{$\begin{array}{l}\text { Time } \\
\text { or } \\
\text { Profile }\end{array}$} & \multicolumn{5}{|c|}{ Infrared Flux (W $\left.\mathrm{m}^{-2}\right)$} \\
\hline & $\begin{array}{l}\text { Out- } \\
\text { going }\end{array}$ & $\begin{array}{c}\text { Outgo } \\
\text { from } \\
\text { Surface }\end{array}$ & $\begin{array}{l}\text { Down- } \\
\text { welling }\end{array}$ & $\begin{array}{l}\text { Down } \\
\text { from } \\
0.6 \mathrm{mb}\end{array}$ & $\begin{array}{c}\text { Surface } \\
\text { Net }\end{array}$ \\
\hline 07:00 & 279.51 & 38.59 & 361.26 & 94.92 & 19.78 \\
\hline $11: 00$ & 290.71 & 48.92 & 399.14 & 132.32 & 59.76 \\
\hline $15: 00$ & 297.09 & 51.41 & 428.23 & 157.98 & 68.89 \\
\hline 19:00 & 291.50 & 45.89 & 408.85 & 145.11 & 51.03 \\
\hline $23: 00$ & 284.53 & 40.45 & 382.91 & 113.51 & 29.16 \\
\hline 03:00 & 280.77 & 38.68 & 367.64 & 99.32 & 20.57 \\
\hline Mean & 287.62 & 44.31 & 392.05 & 124.17 & 42.95 \\
\hline Mean T\&H & 287.37 & 44.19 & 390.46 & 123.87 & 43.13 \\
\hline Mean T & 287.14 & 48.61 & 385.33 & 116.47 & 48.27 \\
\hline Constant & 292.60 & 52.04 & 404.80 & 138.91 & 64.99 \\
\hline GENESIS & 291.13 & 46.33 & 394.91 & & 53.35 \\
\hline
\end{tabular}
1. The outgoing flux is the outgoing longwave radiation at the top of the atmosphere due to earth's surface and atmospheric emission, and calculated from Eqn. (1) with $l=0$; the contribution of the surface to the outgoing flux is given by the first term
Table 1. The Daily Variation of Infrared Fluxes

in Eqn. (1) with $l=0$; the down-welling flux is by Eqn. (4) with $l=n$; and the contribution to the downward surface radiation from the lowest layer $(0.6 \mathrm{mb})$ is $2 \pi \Delta \nu \sum_{k=1}^{K} \sum_{j=1}^{5} B\left(\nu_{k}, T_{n-i}\right) \epsilon_{n-1, n}\left(\xi_{j}\right) \xi_{j} w_{j}$. The surface net thermal radiation is $F^{\dagger}(n)-F^{\downarrow}(n)$.

The first 6 rows in Table 1 are the simulated fluxes for profiles at four hour intervals. "Mean T\&H" refers to the fluxes calculated from the 24 hour average temperature and water vapor mixing ratio profiles. "Mean T" represents results for the 24 hour average temperature profile but the water vapor mixing ratio profile is generated from the average temperature profile and standard relative humidity for mid-latitude summer. "Constant" indicates the simulated fluxes for the constant lapse rate temperature profile which was used along with the standard relative humidity for midlatitude summer to generate the calculated water vapor mixing profile. "GENESIS" shows the simulated daily mean values using the GENESIS model grid spacing.

Simulations showed that about $30 \%$ of the downwelling longwave flux reaching the surface is emitted by the lowest 0.6 millibar, that is, about 5 meters (Table 1, column 5); and about $50 \%$ of the down-welling flux is emitted by the lowest 3 millibars ( 25 meters). Thus the lowest few millibars are very important to the surface thermal radiation energy budget, especially when the actual profile is different from a constant lapse rate profile. In such a thin layer, its temperature is closely coupled to surface temperature.

For this particular case (Figure 4), the average magnitude of the down-welling flux reaching the surface is $392 \mathrm{~W} \mathrm{~m}^{-2}$, and the daily variation is $67 \mathrm{~W} \mathrm{~m}^{-2}$, which is much greater than the magnitude of the daily variation of outgoing flux $\left(17.6 \mathrm{~W} \mathrm{~m}^{-2}\right)$ (Table 1). The contribution from the lowest 0.6 millibars to the daily variation of the down-welling flux is similar to the daily variation for the total down welling flux. The contribution to the daily variation from the lowest $0.6 \mathrm{mb}$ is 63 $\mathrm{W} \mathrm{m}^{-2}$, or $94 \%$ of the total, which again demonstrates the importance of the near-surface air layer. Note that the diurnal variation from the lowest 3 millibars, 85 $\mathrm{W} \mathrm{m}^{-2}$, is larger than the total variation of the downwelling flux. The bottom layers become warmer and 


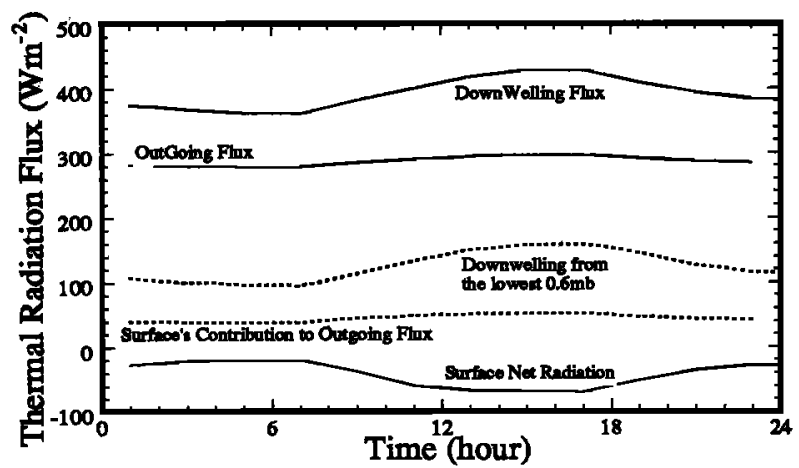

Figure 4. The 24 Hour Variation of the Down-Welling and Outgoing Thermal Infrared Fluxes

moister during the day, and their contribution becomes larger because there is less radiation from above reaching the surface. The opposite is true at night when the bottom layers become cool and contain less water vapor and more radiation from above can reach the surface.

Because the constant lapse rate profile overestimates temperatures and water vapor mixing ratios in the lowest atmosphere, the down-welling flux is overestimated by $12.8 \mathrm{~W} \mathrm{~m}^{-2}$ (Table 1 ), that is, about $3 \%$, and overestimates the contribution from the lowest 0.6 millibars by $14.7 \mathrm{~W} \mathrm{~m}^{-2}$, or, about $11 \%$. The constant lapse rate profile also introduces a large error in the surface net radiation. The surface temperature is overestimated and hence the surface thermal radiation emission and the surface net radiation energy loss. The daily mean value is $43 \mathrm{~W} \mathrm{~m}^{-2}$, while that calculated from the constant lapse rate profile is $65 \mathrm{~W} \mathrm{~m}^{-2}$, with a relative error of more than $50 \%$ and an absolute error of $22 \mathrm{~W} \mathrm{~m}^{-2}$, which is equivalent to a change in surface temperature of $4.0^{\circ} \mathrm{C}$.

The 24 hour variation in the simulated outgoing thermal infrared flux is also shown in Figure 4. It is much less significant than the variation in the down-welling flux. The daily variation is about $18 \mathrm{~W} \mathrm{~m}^{-2}$, about $6.5 \%$ of the average outgoing flux. The constant lapse rate profile overestimates the outgoing flux by $5 \mathrm{~W} \mathrm{~m}^{-2}$, and overestimates the contribution from the surface by $7.7 \mathrm{~W} \mathrm{~m}^{-2}$, about $17 \%$ of the daily average surface contribution.

Most 1980's-vintage climate models do not include diurnal cycles and so do not have a diurnal variation in the long wave radiation, e.g., the various versions of the NCAR CCM version 1. Many 1990's climate models do incorporate diurnal cycles that include the temperature effect on the long-wave radiation; for instance, the GENESIS model [S. L. Thompson and D. Pollard, A Global Climate Model (GENESIS) with a Land-surfacetransfer Scheme, submitted to J. Climate, 1994] does include a diurnal temperature variation in the lowest layer which extends from $\sigma=1.000$ to 0.9585 , but allows water vapor to have only a variation every 24 hours. We have repeated our calculations using this grid spacing and find that the surface net infrared radiation is 53.35 $\mathrm{W} \mathrm{m}{ }^{-2}, 24 \%$ larger in magnitude than that calculated by using a detailed vertical resolution (Table 1).
Although this study was done for a single midlatitude clear sky measurement of temperature, it does demonstrate the importance of the thermal characteristics of the boundary layer to infrared fluxes, especially to the down-welling flux and surface net radiation. Also, because of the daily cycle of solar heating and nocturnal cooling, significant daily variations exist. The diurnal variation is on the order of $10 \%$ for the outgoing flux, about $20 \%$ for the down-welling flux, and greater than $100 \%$ for the surface net thermal radiation.

Acknowledgments. The authors would like to thank D. Portman and H. Marshall from the University of Michigan and D. Pollard from the National Center for Atmospheric Research for constructive suggestions. This work was supported in part by the National Science Foundation, grant number ATM-9105476.

\section{References}

Ellingson, R. G., J. Ellis and S. Fels, The intercomparison of Radiation Codes Used in Climate Models: Long Wave Results, J. Geophys. Res., 96, 8929-8953, 1991.

Feigelson, E. M., B. A. Fomin, I. A. Gorchakova, E. V. Rozanov, Yu. M. Timofeyev, A. N. Trotsenko, and M. D. Schwarzkopf, Calculation of Longwave Radiation Fluxes in Atmosphere, J. Geophys. Res., 96, 8985-9001, 1991.

Fels, S. B., J. T. Kiehl, A. A. Lacis, and M. D. Schwarzkopf, Infrared Cooling Rate Calculations in Operational General Circulation Models: Comparison with Benchmark Computations, J. Geophys. Res., 96, 9105-9120, 1991.

Geiger, R., The Climate near the Ground, Translated by Scripts Technica. Inc., Harvard University Press, 1975.

Gol'tsberg, I. A. (Editor), Microclimate of the USSR, Translated from Russian by D. Laderman, B. M. E. Israel Program for Scientific Translations Ltd., 1969.

Gordley, L. L., and J. M. Russell III, Rapid Inversion of Limb Radiation Data Using an Emissivity Growth Approximation, Applied Optics, 20, 807-813, 1981.

Ma, Q., and R. H. Tipping, A far wing line shape theory and its application to the water continuum absorption in the infrared region. I, J. Chem. Phys., 95, 6290-6301, 1991.

Morcrette, J.-J., and Y. Fouquart, On Systematic Errors in Parameterized Calculations of Longwave Radiation Transfer, Q. J. R. Meteorol. Soc., 111, 691-708, 1985.

Tjemkes, S. A., F. T. M. Nieuwstadt, A Longwave Radiation Model for the Nocturnal Boundary Layer, J. Geophys. Res., 95, 867-872, 1990.

Weinreb, M. P., and A. C. Neuendorffer, Method to Apply Homogeneous-Path Transmittance Models to Inhomogeneous Atmospheres, Journal of the Atmospheric Sciences, $30,662-666,1973$.

Wenzhogh Zhao, Applied Research Corporation, NASA Goddard Space Flight Center, Code 913, Greenbelt, MD 20771. William R. Kuhn and S. Roland Drayson, Department of Atmospheric, Oceanic and Space Sciences, University of Michigan, Ann Arbor, MI.

(received December 6, 1993; revised April 4, 1994; accepted April 21, 1994.) 